




\section{LA ALTERNATIVA KANTIANA DE KELSEN: UNA CRITICA}

\section{Introducción}

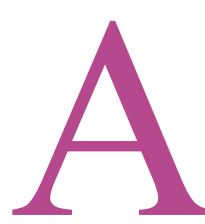

menudo se dice que la Teoría Pura del Derecho de Kelsen es de carácter kantiano o neo-kantiano, y que refleja una kantiana o neo-kantiana «postura intermedia» entre la teoría clásica del Derecho natural de una parte y la teoría del Derecho empírico-positivista de otra. Debe admitirse, que esta «postura cree Kelsen, tiene todos los componentes de la teoría clásica del Derecho natural ${ }^{1}$. Más bien, es un reflejo de diferentes aspectos de la teoría del conocimiento de Kant. Y de una lectura de Kelsen, se desprende que su teoría y la de Kant chocan. Kant resuelve las antinormas matemáticas, planteadas por la yuxtaposición del racionalismo dogmático y el empirismo escéptico, argumentando que la noción de «un mundo de los sentidos que existe por el mismo $»^{2}$-esto es, que existe absolutamente-

${ }^{1}$ «Era sin ninguna duda imposible para una personalidad tan profundamente arraigada en el cristianismo como Kant una completa emancipación de la metafísica. Esto es más evidente en su filosofía práctica: es precisamente aquí, donde se encuentra el énfasis de la doctrina cristiana, cuyo dualismo metafísico invade todo el sistema de Kant, el mismo dualismo contra el que había luchado con determinación en su filosofía teórica. En este momento, Kant ha abandonado su método trascendental, una contradicción en el idealismo crítico que se ha señalado bastante a menudo. Y es de esta forma como Kant, cuya filosofía trascendental estaba destinada a proporcionar, en particular, la fundamentación de una filosofía jurídica y política positiva, permaneció, como filósofo jurídico, en el camino de la teoría del Derecho natural. Ciertamente, su Fundamentación de la metafísica de las costumbres puede considerarse como casi la expresión más perfecta de la teoría del Derecho natural que se desarrolló fuera del cristianismo protestante durante los siglos diecisiete y dieciocho.» H. Kelsen, Die philosophischen Grundlagen der Naturrechtslehre und des Rechtspositivismus, Rolf Heise, Charlottenburg 1928, pág. 76. (La cursiva es de Kelsen.)

${ }^{2}$ E Kant, Prolegómenos, 52 (c). 
equivale a una auto-contradicción y debe ser reemplazada por la noción de que el mundo existe no «por él mismo» sino en relación a la razón ${ }^{3}$. Kant desarrolla esta última posición, su postura intermedia, en la analítica trascendental de La crítica de la razón pura. Así, del mismo modo, se podría argumentar que Kelsen se enfrenta a una «antinomia jurisprudencial». Aunque las tradicionales filosofías del Derecho, la teoría empírico positivista y la teoría del Derecho natural, parecen, cuando se toman juntas, ser exhaustivas con las posibilidades (tertium non datur), ninguna de ellas es defendible. Kelsen resuelve la antinomia demostrando, primero, la posibilidad de una postura intermedia en la filosofía jurídica. Su siguiente paso es desarrollar un caso para esta postura intermedia, y esto por medio de un argumento kantiano ${ }^{4}$.

Si el razonamiento kantiano de Kelsen fuera defendible, su postura intermedia representaría una alternativa real a las teorías tradicionales. De acuerdo con mi posición sobre el razonamiento kantiano de Kelsen respecto a los modelos de argumentación trascendental, esta aproximación kantiana, su postura intermedia, no sólo no le proporciona, sino que, ciertamente, no puede proporcionarle un argumento defendible en apoyo de la Teoría Pura del Derecho. (De esta crítica no se sigue que una postura intermedia sea completamente imposible; tan sólo que un argumento trascendental en apoyo de esta postura intermedia es imposible).

${ }^{3}$ Para una visión general de la primera antinomia matemática, E. Kant, Crítica de la razón pura, A426-438/B454-466, A490-567/b518-595.

${ }^{4}$ William Ebenstein, en su libro The Pure Theory of Law (University of Wisconsin Press, Madison, 1945; reimpresión Rothman, Nueva York, 1969), ofrece la que es quizá la más detallada interpretación kantiana de la Teoría pura de Kelsen en la doctrina. Dentro de la Escuela de Viena la más ambiciosa, aunque breve, teoría kantiana del conocimiento jurídico no se origina en Kelsen sino en su alumno, Fritz Sander. Especialmente remarcable entre los escritos en que Sander desarrolla la teoría es «Die transzendentale Methode der Rechtsphilosophie und der Begriff der Rechtserfahrung», Zeitschrift für offentliches Recht, I (1919-20), 468-507, reimpreso en Sander \& Kelsen, Die Rolle des Neukantianismus in der Reinen Rechtslehre, ed. Stanley L. Paulson, Scientia Verlag, Aalen, 1988, págs. 75-114. Después de haber señalado estas fuentes, debería añadir que no existe acuerdo entre los intérpretes del razonamiento kantiano de Kelsen; al contrario, las interpretaciones en la literatura de la norma fundamental -el instrumento normal para explorar las argumentaciones de Kelsen- se extienden en un amplio espectro, y algunas no tienen en absoluto un componente kantiano (ver la nota 11 más adelante). 


\section{A. La cuestión trascendental}

Un punto de partida útil es la así llamada cuestión trascendental. En la filosofía medieval, los trascendentales (unum, bonum, verum) eran bien conocidos como rasgos generales del ser que trasciende la clasificación entre géneros y especies. Distanciándose radicalmente de esta tradición, Kant en vez de esto retiene algo de su nomenclatura, utilizando el término «trascendental» ${ }^{5}$ para hablar de cognición o conocimiento referido «no tanto a los objetos de conocimiento sino cómo conocemos los objetos, de forma que esto sea posible a priori $\rangle^{6}$. Es esta distinta lectura kantiana de «trascendental»-considerada por Hegel como una «terminología bárbara» y causante, en palabras de Vaihinger, de horribles malinterpretaciones ${ }^{7}$ la que debilita las condiciones de la posibilidad de conocimiento. La cuestión trascendental de Kant se pregunta cómo tal conocimiento o cognición es posible. Kelsen, siguiendo conscientemente a Kant en este particular, sitúa su cuestión trascendental: «¿Cómo es posible el Derecho positivo como objeto de cognición, como objeto de ciencia jurídica cognitiva?» ${ }^{8}$.

Kelsen está buscando un argumento en apoyo de la función constitutiva de la ciencia jurídica cognitiva. Sostiene que la ciencia jurídica se centra en ciertos datos (actos de voluntad), conocidos por medio de una interpretación «objetiva». La ciencia jurídica en relación con esto establece de nuevo los datos sacados de materia prima regulada ${ }^{9}$. Los datos conocidos, «objetivamente»

5 El desarrollo histórico preparando el camino para la recepción por Kant del concepto de «trascendental» lo señala Norbert Hinske en «Die historischen Vorlagen der Kantischen Transzendental-philosophie», Archiv für Bergriffigeschichte, 12 (1968), págs. 86-113, especialmente las págs. 89-95.

${ }^{6}$ E. Kant, Crítica de la razón pura, B25.

${ }^{7}$ Hegel, Lectures on the History of Philosophy, trad. E. S. Haldane \& F. H. Simon, vol. 3, (London: Kegan Paul, Trench, Trübner, 1896), pág. 431: Hans Vaihinger, Commentar zu Kants Kritik der reinen Vernunft, vol. 1:W. Spemann, Stuttgart, 1881, pág. 467.

${ }^{8}$ Philosophische Grundlagen, nota 1, pág. 66.

${ }^{9}$ En este aspecto de la alternativa kantiana de Kelsen, es decir, la noción de la función constitutiva de la ciencia jurídica cognitiva, hay algún acuerdo entre los contemporáneos de Kelsen, ver por ejemplo Franz Weyr, «Reine Rechtslehre und Verwaltungsrecht», en Gesellschaft, Staat und Recht, ed, Alfred Vedross, Springer, Viena, 1931, págs. 366-89, 370-72 y 375. También entre la reciente doctrina, ver por ejemplo, Wolfgang Schluchter, Entscheidung für den sozialen Rechtsstaat, Kiepenheuer \& Witsch, Cologne, 1968 (reimpresión Nomos, Baden-Baden, 1983), págs. 27-32. Las dificultades y la correspondiente falta de acuerdo aparecen cuando uno se pregunta por qué la ciencia jurídica debería tener una función constitutiva; me ocuparé del asunto más adelante. 
interpretados ahora toman la forma de normas jurídicas hipotéticamente formuladas o reconstruidas $^{10}$ que constituyen los apropiados objetos de conocimiento de la ciencia jurídica.

Al formular su cuestión trascendental, Kelsen no está preguntando si nosotros conocemos el material jurídico, o si conocemos que ciertas proposiciones jurídicas son verdaderas. Ciertamente, él supone que tenemos tal conocimiento y se pregunta cómo lo hemos adquirido. Esto capta algo del peculiar desarrollo trascendental de la cuestión de Kelsen: dado que conocemos algo que es verdad, ¿qué presunción le afecta? Específicamente, ¿qué presunción le afecta y sin la cual la proposición que nosotros sabemos que es verdad podría no serlo?

\section{B. La norma fundamental y el argumento trascendental}

El intento de Kelsen de responder a la cuestión trascendental depende de la referencia a la norma fundamental ${ }^{11}$. Es clara la idea intuitiva que sigue a la norma fundamental. Kelsen defiende en todo su trabajo una fuerte y firme distinción entre «es» y «debe», una distinción bien conocida del dualismo metodológico de los neo-kantianos de Heidelberg ${ }^{12}$ y, al modo de la tesis normativa, defendida por Kelsen en su Teoría Pura. La distinción entre «es» $\mathrm{y}$ «debe» implica enteramente caminos separados para establecer, respectivamente, la verdad de las pretensiones empíricas y, inter alia, la validez de las normas jurídicas ${ }^{13}$. Naturalmente,

${ }^{10}$ Ver Kelsen, Reine Rechtslehre, Deuticke, Leipzig \& Viena, 1934, § 11 (b).

${ }^{11}$ Ver Philososphische Grundlagen, nota 1, pág. 66. Sobre la norma fundamental ver Kelsen, Reine Rechtslehre (nota 10), §§ 27-31 (a). Se han ofrecido por la doctrina, gran cantidad de interpretaciones diferentes variando desde una aproximación trascendental kantiana (discutida más adelante), a la noción de Alfred Schutz de la noción de norma fundamental como principio para la construcción de esquemas interpretativos ideales-típicos, o a la aproximación de Robert Walter en términos de una suposición «como si» no distinta de la de Vaihinger. Ver Alfred Schutz, The Phenomenology of the Social World (primera edición de 1932), traducción G. Walsh \& F. Lehner, Northwestern U. P., Evanston, III. 1967, págs. 246-48; Robert Wafter, «Der gegenwärtige Stand der Reinen Rechtslehre», Rechtstheorie, I (1970), págs. 69-95. Especialmente en págs. 73 y $80-83$.

${ }^{12}$ Ver, por ejemplo, Gustav Radbruch, Reschtsphilosophie, 8. Aufl. Ed. Erik Wolf, K. F. Koehler, Stuttgart, 1973, §2, págs. 93-101.

${ }^{13}$ Unir la distinción entre «es» y «debe» a la tesis normativa, por medio del dualismo metodológico, no es negar la conocida distinción entre «es» $\mathrm{y}$ «debe» asociada a la tesis de la separabilidad. Al contrario, Kelsen defiende ambas. Pero la distinción entre «es» y «debe» es sistemáticamente ambigua. Cuando se 
el interés de Kelsen se centra en esto último. Desde su punto de vista, la validez de una norma jurídica se establece en referencia a la apropiada norma de nivel superior, cuya propia validez se establece, sucesivamente, con referencia a la correspondiente norma de nivel superior a ella, y así sucesivamente, hasta que se alcanza la norma de nivel más alto en el sistema jurídico, el nivel de la constitución ${ }^{14}$. Más allá del nivel constitucional, no puede haber más referencias. Una referencia a un nivel todavía más alto de normas de Derecho positivo se excluye ex hypothesi. Y una referencia a algún tipo de hecho está cerrada por la fuerte y firme distinción entre «es» y «debe», como reflejo del dualismo metodológico. Una tercera vía de referencia, la de la moral, está cerrada por la tesis de la separación.

¿Cómo se establece, entonces, la validez de las normas en el nivel constitucional? A falta de ninguna otra referencia, se asume su validez. Y la asunción toma la forma de la norma fundamental.

Esta lectura de la norma fundamental -o, más precisamente, de la idea intuitiva causante de la norma fundamental- es evidente en una de las más tempranas expresiones de Kelsen como de la «norma más elevada» o la «norma última», como la llamó posteriormente $^{15}$. De esta forma, sin embargo, la referencia a la norma fundamental claramente utiliza un razonamiento circular. Ya que si el problema es porqué las normas del nivel más alto son válidas, y luego simplemente se asume que son válidas, nos coloca de nuevo ante el mismo problema.

invoca en nombre de la tesis normativa, el auxiliar modal «debe» se refiere a normas jurídicas, y el «es» da la expresión a los hechos. Invocado en nombre de la tesis de la separabilidad, «debe» se refiere a normas de moralidad» y «es» expresa lo válido, esto es normas jurídicas existentes. (Para la concepción de normatividad en Kelsen ver Reine Rechtslehre (nota 10), en el § 16.

${ }^{14}$ Ver Kelsen, Reine Rechtslehre (nota 10), $\S \S 27-28,31$ (a). La idea de encontrar el origen a través de una jerarquía normativa para establecer la validez jurídica es, por supuesto, bien conocida; ver, por ejemplo, James Bryce, Studies in History and Jurisprudence, Oxford U. P., Londres, 1901 , págs. 505-506, y H. L. A. Hart, The Concept of Law, Clarendon Press, Oxford, 1961, págs. 103-104. La «búsqueda del origen» en Kelsen refleja su uso del Stufenbau o estructura jerárquica en la forma desarrollada por su compañero en la Escuela de Viena, Adolf Merkl. Para un resumen del Stufenbau de Merkl con otros puntos de vista sobre la noción de Robert Walter, Theo Öhlinger, Werner Krawietz y Ota Weinherger, ver Jurisprudence in Germany and Austria. Selected Modern Themes, ed. S. L. Paulson, Clarendon Law Series (en prensa).

${ }^{15}$ Ver Kelsen, «Reichsgesetz und Landesgesetz nach österreichischer Verfassung», Archiv des öffentlichen Rechts, 32 (1914), págs. 202-245. 390-438. Especialmente las páginas 215-20. 
En la segunda y tercera fase de $\operatorname{Kelsen}^{16}$, donde los elementos kantianos y neo-kantianos informan el conocimiento de la norma fundamental, él no proporciona una expresión completa de la noción. Es una noción que necesita clarificación.

La táctica que me gustaría seguir en un esfuerzo por entender la norma fundamental y los argumentos filosóficos de la teoría de Kelsen es la de centrarme en el argumento trascendental, un argumento implícito en la norma fundamental- implícito, en cualquier caso, donde la norma fundamental se ofrece como respuesta a la cuestión trascendental de Kelsen. En esta aproximación Kelsen tendría que introducir 1), como su categoría fundamental, la noción de imputación normativa. Luego él mencionaría 2) un razonamiento neo-kantiano o trascendental demostrando esta categoría fundamental como una presunción de los datos que son dados. La norma fundamental sería relegada a un papel subsidiario, porque se haría referencia a ella de un modo similar a cualquier otro tipo de alusión en el curso del trabajo. Desarrollaré brevemente estos dos puntos como un resumen del razonamiento trascendental de Kelsen, luego me centraré en unos detalles del argumento trascendental y finalmente, en una evaluación de las consecuencias de la argumentación para Kelsen así interpretado.

Kelsen introduce la categoría de imputación normativa por analogía con la categoría de causalidad:

Tal como la ley de la naturaleza une a cierto hecho material como causa otro como efecto, así de igual manera la norma jurídica une la condición jurídica con la consecuencia jurídica (la consecuencia de un llamado acto ilegal). Si la forma de unir los hechos es en un caso la causalidad, en el otro lo es la imputación ${ }^{17}$.

Una aproximación inicial a un razonamiento trascendental,

${ }^{16}$ No existe una completa periodificación de la obra de Kelsen. Lo que yo tengo en mente puede ser resumido en cuatro fases: una primera, la fase «constructivista», evidente en el Habilitationsschrift de 1911, una fase que desplaza el rumbo de la siguiente década por la desviación de Kelsen, aunque de forma irregular, hacia preceptos neo-kantianos. Después de 1934, empezó a introducir conceptos del repertorio empirista, manejando en alguno de sus escritos, por ejemplo, la noción de causalidad de Hume y añadiendo que una categoría a priori de causalidad sería un paso en una dirección errónea, apartándose de Hume. Finalmente, después de 1960, Kelsen colocó encima de su segunda y tercera fases la Teoría Pura del Derecho tal y como la conocemos, introduciendo elementos de la teoría de la voluntad para reemplazarlas.

${ }^{17}$ Kelsen, Reine Rechtslehre (nota 10), § 11 (b). 
incorporando la categoría de imputación normativa en su segunda, premisa «trascendental», podría explicarse como sigue:

\section{Razonamiento I}

1. Se tiene conocimiento de las normas jurídicas (dadas).

2. El conocimiento de las normas jurídicas sólo es posible si se presupone la categoría de imputación normativa (premisa trascendental).

3. Por consiguiente, la categoría de imputación normativa se presupone (conclusión trascendental).

Será útil, después, ver el papel que juega el escéptico y, la distinción entre las versiones progresiva y regresiva del razonamiento trascendental. Esto requiere volver atrás de esta aproximación inicial y centrarse en una caracterización más abstracta del argumento.

C. Estructura del argumento trascendental: el papel del escéptico, versiones progresiva y regresiva

Una caracterización abstracta del argumento trascendental ${ }^{18}$, podría empezar con la afirmación $P$, asumida como verdadera, la cual puede ser verdad sólo si otra afirmación $Q$, es también verdadera. Esto es,

\section{Razonamiento II}

1. $P$.

2. 'P' es posible sólo si $Q$.

3. Entonces $Q$.

${ }^{18}$ Esto lo he extraído de la considerable literatura existente sobre los argumentos trascendentales, y mucho de ello se ha inspirado en el trabajo de Peter Strawson. Ver Individuals, Methuen, Londres, 1959, págs. 34-36 y The Bounds of Sense, Methuen, Londres, 1966, págs. 15-32, 72-74, 85-89 et passim. Para una lúcida argumentación en pocas palabras ver Ralph C. S. Walker. Kant, Routledge \& Kegan Paul, Londres, 1978, págs. 9-27. Ver también Transcendental Arguments and Science, ed. Peter Bieri y otros, Reidel, Dordrecht \& Boston, 1979; New Perspectives on Transcendental Arguments and Critical Philosophy, ed. Eva Schaper \& Wilheim VossenkultI, BlackweIl, Oxfor, 1989, y -un completo tratado- Reinhold Aschenberg, Sprachanalyse und Transcendental philosophie, Klett-Cotta, Stuttgart, 1982. 
En la teoría del conocimiento de Kant, $P$ representa las impresiones dadas a la conciencia, y $Q$-después de un número de pasos intermedios- representa la categoría kantiana aplicable (más claramente la categoría de causalidad). Una vez que se llegue a $Q$ (en la línea 3 del razonamiento) ${ }^{19}$, entonces -después de unos pasos intermedios- se podrían extraer algunas consecuencias adicionales (demostrando, en la fraseología kantiana, las leyes de la naturaleza como proposiciones sintéticas a priori). Estas consecuencias adicionales son representadas por $R$ en el esquema del argumento. Esto es,

\section{Por consiguiente $R$.}

El uso del argumento de Kant en La crítica de la razón pura se señala como una respuesta al escéptico -el desafío escéptico al argumento de Kant fue proporcionado por el filósofo David Hume y, en la filosofía jurídica, la posición del anarquista como escéptico fue sugerida por el propio Kelsen ${ }^{20}$. Ésta es la fuerza del argumento de Kant. Demuestra, si está bien fundamentado, que el escéptico no puede ayudar sino debilitar su propia posición al intentar defenderla.

La estrategia de Kant es disponer los pasos del razonamiento de forma que atrape al escéptico. Esto es, Kant introduce datos

${ }^{19}$ Desde el punto de vista de la validez lógica, el argumento no es problemático; completado con una línea adicional

1 a) Si $P$, entonces 'P' es posible

el razonamiento sigue, a través de la regla de la inferencia modus ponens, en un camino sin dificultades a la conclusión en la línea 3. Bastante más difícil es la cuestión de la posición lógica de las premisas, en particular, la propia versión de Kant de la línea 2. Algunos escritores insisten en que esta premisa tiene que ser analítica, mientras otros oponen que tienen que ser entendida propiamente como sintética a priori. Ver por ejemplo, Walker, Kant (nota 18), págs. 18-22, defendiendo la primera posición en respuesta a una defensa de la última posición por T. E. Wilkerson, «Transcendental Arguments», Philosophical Quarterly, 20 (1970), págs. 200-12, y Wilkerson, Kants Critique of Pure Reason, Clarendon Press, Oxford, 1976, págs. 202-206. Afortunadamente, podemos ignorar esta difícil cuestión aquí, ya que tal como argumentaré aunque la versión del trascendental de Kant está fundada, la característica versión neokantiana del argumento no lo está.

${ }^{20}$ Ver el texto citado en la nota 24 más adelante. Mientras reconoce claramente al anarquista como escéptico en relación con la teoría normativa jurídica, Kelsen es igualmente claro (como podremos ver) en rechazar la sugerencia de que la Teoría Pura podría ser construida como una respuesta al escéptico. Si algo queda de la versión regresiva del argumento trascendental una vez que ha sido hecha esta concesión es una cuestión de la que me ocuparé más adelante. 
en la primera premisa que necesariamente el escéptico considerará necesarios para su propia posición, pero tan pronto como el escéptico esté de acuerdo con la primera premisa, la transición a la categoría aplicable de Kant es inevitable y el pobre escéptico es cazado. Mientras que podía haber estado completamente de acuerdo con la primera premisa, nunca habría soñado con estarlo con el resto del razonamiento, ya que la categoría kantiana y, en particular, las conclusiones adicionales derivadas de ella es de lo que él era escéptico. Como Ross Harrison señala espléndidamente, refiriéndose al dilema del escéptico:

«Si el escéptico no puede decir nada importante sin presuponer la verdad de la cosa sobre la que está dudando o negando, entonces debe hacer una elección entre el silencio y la derrota» ${ }^{21}$.

Si una clave en la estructura del razonamiento trascendental es el papel jugado por el escéptico, otra es la distinción bien conocida desde los Prolegómenos de Kant, entre la versión progresiva y regresiva del razonamiento ${ }^{22}$. El Razonamiento I, descrito con anterioridad, refleja la versión regresiva del razonamiento trascendental, punto sobre el cual volveré. El Razonamiento II, descrito inmediatamente después, ilustra la versión progresiva, reflejando la clase de razonamiento (aunque extremadamente condensado) que Kant o el filósofo kantiano podría desarrollar en nombre de la «deducción trascendental de las categorías» en La crítica de la razón pura $^{23}$. La versión progresiv

${ }^{21}$ Ross Harrison, «Wie man dem transzendentalen ich einen Sinn verleilit», trad. Woligang R. Köhler, en Kants transzendentale Deduktion und die Möglichkeit von Transzendentalphilosophie, ed. Forum für Philosophie Bad Homburg, Suhrkamp, Frankfurt, 1988, págs. 32-50, especialmente págs. 34-35.

${ }^{22}$ E. Kant, Prolegómenos, $§ 5$ (nota):

«(el) método analítico... supone que se empieza desde lo que está siendo buscado como si fuera dado, y se asciende hasta las condiciones bajo las cuales es posible. En este método a menudo se usan solamente proposiciones sintéticas, como en el ejemplo del análisis matemático, por lo que podría ser mejor llamarlo método regresivo en contraposición al método sintético o progresivo».

Significativamente, Kant usa el modo subjuntivo («como si fuera dado») en sus líneas en el así llamado método regresivo, sugiriendo que este método es una mera formulación sumaria de los problemas que esperan demostración por medio del método progresivo. Por otra parte, los neo-kantianos, toman el «hecho (faktum) de la ciencia» como algo que es realmente dado (ver el texto citado en la nota 28), en contraste con Kant ellos están usando el método regresivo independientemente del método progresivo.

${ }^{23}$ Se podría hacer una cautelosa aproximación al argumento de Kant hablando a través del «filósofo kantiano» más que del Kant histórico. No existe 
comienza de forma débil para ganar el inmediato acuerdo del escéptico y luego prosigue para demostrar la aplicación de las categorías como una condición de la posibilidad real de la experiencia. La clave, al igual que con la versión progresiva del razonamiento, está en el papel jugado por el escéptico.

\section{Kelsen y la versión progresiva del razonamiento trascendental.}

Kelsen deja claro que no sigue lo que he descrito como la versión progresiva del argumento trascendental. Tal como lo señala en el presente texto:

«Es bien sabido de la teoría pura que uno no puede probar la existencia de una norma como prueba la existencia de los hechos de la naturaleza y las leyes naturales que los gobiernan, que uno no puede refutar con argumentos irrefutables una postura como el anarquismo teórico, la cual rechaza ver otra cosa excepto el crudo poder donde los juristas hablan de Derecho» ${ }^{24}$.

Un cuarto de siglo más tarde, Kelsen se refiere al mismo tema y trata la misma cuestión:

«Uno puede distinguir entre una norma obligatoria justa e injusta e interpretar objetivamente las relaciones interpersonales, como relaciones jurídicas, específicamente, como deberes jurídicos, derechos, y poderes, sólo si uno presupone esta (categoría de imputación normativa). Todavía, esto es sólo una posible interpretación, hecha posible por la presunción de la (categoría) y dependiente de ella; no es una interpretación necesaria. Las relaciones interpersonales pueden también ser interpretadas como meras relaciones de poder, esto es, como causas y efectos, siguiendo la ley de la causalidad» ${ }^{25}$.

un acuerdo sobre la evolución de «la deducción trascendental de las categorías» de Kant ni, ciertamente, un razonamiento detallado de lo que, en este contexto, quiere decir la expresión jurídica «deducción» en el siglo dieciocho de Kant. (De esto último ver Dieter Henrich, «Kant's notion of a deduction and the Methodological Background of the First Critique», en Kants Transcendenta Deductions, ed. Eckar Fórster, Stanford U. P., Stanford, 1989, págs. 29-46). Mi uso del razonamiento de Kant gira en torno, sin embargo, sólo a la interpretación neo-kantiana de él (ver nota 19); en su interpretación del razonamiento, a saber, como regresivo en carácter, hay algo de aproximación a un acuerdo (ver la nota 27 más adelante).

${ }^{24}$ Kelsen, Reine Rechtslehre (nota 10), § 16.

${ }^{25}$ Kelsen, «On the Basis of Legal Validity» (primera publicación en 1960), trad. Stanley L. Paulson, American Journal of Jurisprudence, 26 (1981), págs. 
En resumen, expresamente admite la posición del escéptico normativo o del empirista jurídico como una alternativa a su propia posición. Mientras esta admitida alternativa, sin duda, no se considere como otra teoría normativa del Derecho, que ofrezca otros medios que proporcionen coherencia a los datos en cuestión. Es precisamente tal alternativa, sin embargo, la que la versión progresiva del razonamiento trascendental excluiría, si estuviese bien fundada.

La versión regresiva del razonamiento trascendental parecería ajustarse mejor por consiguiente a las intenciones de Kelsen. Y, siguiendo el ejemplo de la tradición neo-kantiana (de la que me ocupo en el curso de la siguiente sección), uno puede entender razonablemente la teoría de Kelsen tomando su apoyo en la versión regresiva.

\section{E. Kelsen y la versión regresiva del argumento trascendental}

La versión regresiva del razonamiento trascendental se explicó anteriormente en el Razonamiento I. Se entiende mejor por contraste con la versión progresiva, para la versión regresiva, el filósofo kantiano le da la vuelta a la versión progresiva. Esto es, lo que se derivaba de la versión progresiva del razonamiento como conclusión secundaria -representada por R en el Razonamiento II- se toma como el punto de partida de la versión regresiva.

Versión progresiva

Razonamiento II (modificado del anterior) ${ }^{26}$.

1. $P$ (datos de conciencia dados).

2. ${ }^{*} P \rightarrow Q$ (categoría como condición).

3. $Q$ (categoría como conclusión).

178-89, concretamente en las págs. 185-86 (la cursiva es de Kelsen). Para otras referencias a Kelsen en el mismo punto ver Raz, The Authority of Law; Clarendon Press, Oxford, 1979, págs. 135-38.

${ }^{26} \mathrm{El}$ símbolo * en la línea 2 de cada razonamiento expuesto representa aquí el denominado operador modal de posibilidad; el símbolo toma el puesto de la formulación «es posible que» en las primeras proposiciones de estos razonamientos. 




En contraste con el débil punto de partida de la versión progresiva, que toma como dados los datos de la conciencia, el punto de partida de la versión regresiva es más fuerte ya que toma como dado algo ya conocido realmente. La afirmación $R$ es una afirmación de conocimiento. El escéptico no prestará su consentimiento aquí, efectivamente, porque es precisamente tal pretendido conocimiento el que es el objetivo de su escepticismo. Pero Kelsen no tiene en cuenta esto, señalando expresamente que no quiere de ningún modo responder al escéptico.

La versión regresiva del razonamiento trascendental, tratada por Kant en sus Prolegómenos como un mero resumen de la versión progresiva, emerge como una forma característica de razonamiento entre los neo-kantianos, y en el no menor de todos ellos Hermann Cohen, la primera figura de la Escuela de Marburg ${ }^{27}$. En una exposición general del método trascendental kantiano, Cohen escribe:

${ }^{27}$ Sigo la admitida opinión en relación con el empleo de Cohen de la versión regresiva del razonamiento trascendental en su interpretación de Kant. Se reconoce en passend por ejemplo, por Rüdiger Bittner, Trascendental Arguments (nota 18), pág. 32, en respuesta a Manfred Baum, ibid, págs. 1-7; por Wolfgang Carl, "Kant's First Drafts of the Deduction of the Categories», en Kant's Transcendental deductions Z (nota 23), págs. 3-20, específicamente, págs. 9-10; y por Hans-Georg Gadamer, «Philosophy or Theory of Science?», en Reason in the Age of Science, tr. Frederick G. Lawrence, MIT Press, Cambridge, Mass., 1981, págs. 151-69, especialmente en las págs. 151-52. Para una discusión centrada en el uso de Cohen de la versión regresiva, ver Manfred BAUM, «Transzendentale Methode», en Historisches Wörierbuch der Philosophie, vol. 5, Schwabe, Babel, 1980, págs. 1375-78, reimpreso en Deduktion und Beweis in Kants Transzendentalphilosophie, Athenäum, Königstein, 1986, págs. 213-18; Aschemberg, Transzenden tal philosophie (nota 18), págs. 367-69; Gcert Edel, Von der Vernunftkrilik zur Erkenninislogik, Karl Alber, Freiburg \& Munich, 1988, págs. 86-88 y 100-145. Finalmente, debería mencionarse que 
«Si... yo tomara el conocimiento no como una forma y manera de la conciencia sino como un hecho que se ha establecido por él mismo en la ciencia y que continúa estableciéndose el mismo en las fundamentaciones dadas, entonces la pregunta no se dirige a un hecho subjetivo sino a un hecho que cualquiera que sea el alcance de su auto-propagación, es no obstante un hecho objetivamente dado, un hecho basado en principios. En otras palabras, la cuestión ya no se dirige al proceso y a la estructura de conocimiento, sino a su resultado, a la misma ciencia. Entonces la pregunta aparece inequívocamente: ¿De qué presunciones deriva su certeza este hecho científico?» ${ }^{28}$.

Cohen deja clara en esta y otras explicaciones del método trascendental de $\mathrm{Kant}^{29}$ que el razonamiento consiste en proceder regresivamente o «hacia atrás»-de la experiencia que ya es conocida, del Faktum de la ciencia, a las categorías presupuestas o principios. Kelsen interpreta el método trascendental de Kant de la misma forma ${ }^{30}$.

$\mathrm{Si}$ seguimos el razonamiento regresivo de Kelsen como se bosqueja en el Razonamiento I, de arriba, confrontamos cuestiones procedentes de la problemática segunda premisa del razonamiento. Kelsen exige allí que la posibilidad real de conocimiento de las normas jurídicas presuponga la aplicación de una categoría de imputación normativa. Pero ¿es sostenible tal pretensión, incluso si fuera prima facie? ¿No existe otra manera de asegurar una filosofía jurídica que lleve juntas la tesis normativa y la tesis de la separabilidad?

Para someter la exigencia de Kelsen a prueba, se puede buscar o una aproximación por etapas para establecer la exigencia o un razonamiento demostrativo. La aproximación por etapas, intentando anticipar todas las posibles teorías que compiten para demostrar que cada una de ellas es indefendible, no ofrece obviamente

la interpretación kantiana de Cohen en este punto ha sido seguida generalmente por los neo-kantianos; ver, por ejemplo, Bruno Bauch, Wahrheit, Wert una Wirklichkeit, Felix Meiner, Leipzig, 1923, pág. 360; Ernst Cassirer, The Problem of Knowledge, tr. William H. Woglom y Charles W. Hendert, Yale U. P., New Haven, 1950, pág. 14.

${ }^{28}$ Hermann Cohen, Des Prinzip der Infinitessimalmethode und seine Geschichte, la primera edición es de 1883, Suhrkamp, Frankfurt, 1968, págs. 4748. (La cursiva es de Cohen).

${ }^{29}$ Ver, por ejemplo, H. Cohen, Kants Begründung der Ethik, Ferd. Dümmler, Berlín, 1877, págs. 24-25 et passim, Kants Theorie de Erfahrung, 2 ed. Ferd. Dümmler, Berlín, 1885, pág. 77.

${ }^{30}$ Ver Kelsen, «Rechtswissenschaft und Recht», Zeitschrift für öffentliches Recht, 3 (1922), pág. 128 et passim, reimpreso en Sander \& Kelsen, Die Rolle des Neukantianismus (nota 4), pág. 304. 
una segura exhaustividad. Agotar las posibilidades requiere, en cambio, un razonamiento demostrativo. Los kantianos dirían, sin embargo, que un razonamiento demostrativo, excluiría de un sólo golpe todas las posibles alternativas a la categoría de Kelsen de imputación normativa, equivalente a la versión progresiva del razonamiento trascendental. Como hemos visto, Kelsen no tenía en mente una versión progresiva de razonamiento. Pero ¿cómo le iría si se dirigiera hacia la versión progresiva, como parece que hace?

No le iría muy bien. La premisa inicial de la versión progresiva del razonamiento trascendental tiene que ser débil, como hemos visto, y si no el escéptico no estará de acuerdo con ella. Más específicamente, como el escéptico no necesita estar de acuerdo con ninguna interpretación de la experiencia dada (él tendrá siempre una interpretación alternativa), entonces para ganar su favor con respecto a la premisa inicial, con la idea de atraparle, esta premisa debe recoger datos que estén bajo el umbral de la experencia interpretada. Pero, precisamente porque están bajo el umbral, estos datos se prestarán a una variedad de diferentes interpretaciones. Y -volviendo ahora al contexto jurídico- el escéptico no tendrá una razón que le obligue a asentir la interpretación normativa de los datos, mucho menos a una interpretación normativa en la forma sugerida por Kelsen.

En una palabra, el escéptico normativo o el empirista jurídico pueden fácilmente dar su aprobación a la primera premisa de la versión progresiva del razonamiento trascendental sin estar obligados por ello a nada más. El razonamiento trascendental, diseñado para «comprometer» al escéptico a cruzar la línea una vez que haya aceptado la premisa inicial, tiene entonces que fracasar.

En resumen, Kelsen se enfrentaría a un problema con cualquier versión del razonamiento trascendental. La versión progresiva toma como su punto de partida una premisa que es simplemente demasiado débil para ser de alguna ayuda al filósofo del Derecho cuando se encuentra con el desafío del escéptico, por lo que parece inútil esta versión del razonamiento trascendental. Este es el punto esencial. Volviendo a la versión regresiva del razonamiento trascendental, más próxima para el filósofo del Derecho, su segunda premisa exige demasiado, como si la única forma de apoyar una teoría normativa del Derecho fuera por medio de la categoría de la imputación. Más precisamente, la segunda premisa exige demasiado a menos que pueda apoyarse en la versión regresiva. La cual nos lleva a un círculo cerrado, con una vuelta a la impracticable versión progresiva. Y la conclusión 
es ineludible: para el filósofo del Derecho, ninguna versión del razonamiento funciona.

Que Kelsen no tuvo intención de usar la versión progresiva sólo pone de relieve aquí el problema más general, que es el proceder como si la versión regresiva del razonamiento pudiera ser utilizada sola, separada de la versión progresiva. Esta separación de la versión regresiva, un movimiento característico de los neo-kantianos, le quita al argumento su fuerza trascendental. El razonamiento es prometedor sólo si ofrece una respuesta favorable al escéptico. Y la versión regresiva del razonamiento puede hacer eso sólo si se entiende como la suplencia de un razonamiento trascendental bien fundado en la versión progresiva. Una vez que las dos versiones sean rigurosas, como en el esfuerzo de los neo-kantianos de extender el razonamiento a varios campos específicos ${ }^{31}$, se pierde el peculiar elemento trascendental y la versión regresiva aparece propiamente como un esquema de análisis.

Podría concluir con una sola observación, sugerida al examinar la alternativa kantiana de Kelsen. En primer lugar, incluso si la llamada versión regresiva del razonamiento trascendental se derrumba en un mero esquema de análisis, de aquí no se sigue que la Teoría Pura del Derecho de Kelsen también lo haga. Más bien, la teoría debe simplemente situarse al lado de otras teorías normativas del Derecho, sujetándose a examen igual que otras teorías. ¿Qué habrá cambiado, sí la Teoría Pura es examinada de esta manera? ¿Su exigencia de unicidad, su exigencia de ser la única posible contendiente para una teoría normativa del Derecho (sans Derecho natural)? Esa exigencia -Inspirada por un razonamiento trascendental que no puede funcionar- debe ser inmediatamente abandonada.

(Trad. José García Añón)

31 Ver, por ejemplo, Georg Simmel, quien en su Soziologie considera la cuestión trascendental: ¿Cómo es posible la sociedad?, y enlaza la investigación con la epistemología de Kant (aunque no, efectivamente, sin importantes distinciones); G. Simmel, Soziologie, Duncker \& Humblot, Leipzig, 1908, págs. 27-45. 